\title{
ADHD symptoms in the adult general population are associated with factors linked to ADHD in adult patients
}

Ting Li $\mathrm{MD}^{\mathrm{a}}$, Nina Roth Mota $\mathrm{PhD}^{\mathrm{a}, \mathrm{b}}$, Tessel E. Galesloot $\mathrm{PhD}^{\mathrm{c}}$, Janita Bralten $\mathrm{PhD}^{\mathrm{a}}$, Jan K.

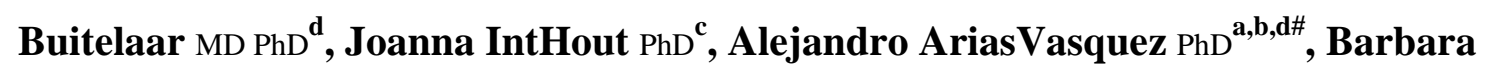
Franke $\mathrm{PhD}^{\mathbf{a}, \mathbf{b}, \mathbf{d} \# *}$

a Department of Human Genetics, Donders Institute for Brain, Cognition and Behaviour, Radboud University Medical Center, Nijmegen, The Netherlands

${ }^{\mathbf{b}}$ Department of Psychiatry, Donders Institute for Brain, Cognition and Behaviour, Radboud University Medical Center, Nijmegen, The Netherlands

${ }^{\mathbf{c}}$ Radboud Institute for Health Sciences, Radboud University Medical Center, Nijmegen, The Netherlands

${ }^{\mathbf{d}}$ Department of Cognitive Neuroscience, Donders Institute for Brain, Cognition and Behaviour, Radboud University Medical Center, Nijmegen, The Netherlands

\#shared final responsibility

*Correspondence to: Department of Human Genetics (855), Radboud University Medical Center, PO Box 9101, 6500 HB Nijmegen, The Netherlands. E-mail address:

barbara.franke@radboudumc.nl (B. Franke) 


\begin{abstract}
Attention-deficit/hyperactivity disorder (ADHD) is a neurodevelopmental disorder in children and adults. It is characterized by inappropriate levels of inattention (IA) and/or hyperactivity and impulsivity (HI). The ADHD diagnosis is hypothesized to represent the extreme of a continuous distribution of ADHD symptoms in the general population. In this study, we investigated whether factors linked to adult ADHD as a disorder are associated with adult ADHD symptoms in the general population. Our population-based sample included 4,987 adults (mean age 56.1 years; 53.8\% female) recruited by the Nijmegen Biomedical Study (NBS). Participants completed the Dutch ADHD DSM-IV Rating Scale for current and childhood ADHD symptoms, the Symptom Check List-90-R (SCL-90-R) anxiety subscale, and the Eysenk Personality Questionnaire (EPQR-S). Partial Spearman correlation and Hurdle negative binomial regression analysis were used to assess how age, sex, childhood ADHD symptoms, anxiety symptoms, and personality traits (neuroticism, extraversion, and psychoticism) are associated with current IA and HI symptoms. Increasing age was associated with a lower proportion of participants reporting HI symptoms and with reduced levels of HI; IA levels remained fairly stable over the age-range, but the probability of reporting IA symptoms increased throughout middle/late adulthood. Females were more likely to report IA symptoms than males. Childhood ADHD symptoms, neuroticism, and psychoticism were positively associated with current IA and HI symptoms, while extraversion had an opposite association with these symptom domains. Anxiety symptoms affected HI symptoms in females. Our results indicate that factors associated with categorical ADHD are also correlated with ADHD symptoms in the adult population.
\end{abstract}

Keywords: ADHD symptomatology, Personality traits, Anxiety, Hurdle negative binomial regression 


\section{Introduction}

Attention-deficit/hyperactivity disorder (ADHD) is a prevalent neurobehavioral disorder in children as well as in adults (Faraone et al., 2015). Meta-analyses of epidemiological studies estimate the prevalence of ADHD to be around 5.3\% to 7.2\% in childhood/adolescence and 2.5\% to 4.4\% in adulthood (Kessler et al., 2006; Polanczyk et al., 2007; Simon et al., 2009; Thomas et al., 2015). ADHD is characterized by developmentally inappropriate, increased levels of two symptom domains, inattention (IA) and/or hyperactivity and impulsivity (HI) (APA, 2013), which manifest themselves during childhood. The diagnosis, according to the criteria established in the Diagnostic and Statistical Manual of Mental Disorders $5^{\text {th }}$ edition (DSM-5) or the International Classification of Diseases and Related Health Problems (ICD10) (APA, 2013; WHO, 1992), uses a categorical definition based on the number of symptoms reported by patients and/or their parents, with functional impairment being an important additional criterion. In the clinical setting, ADHD shows the following main presentations: predominantly inattentive symptoms, predominantly hyperactive-impulsive symptoms, and a combined presentation (having high symptoms counts in both symptom domains).

As a multifactorial disorder, ADHD is influenced by genetic and environmental factors, as well as the interplay between these factors (Faraone et al., 2015). It has a well-recognized genetic vulnerability, with heritability estimates of 70-80\% in both children and adults (Faraone et al., 2015). In addition to genetic susceptibility, factors such as age, sex, psychiatric comorbidities, and personality traits also influence the development of ADHD and its clinical manifestation (Costello et al., 2003; Kessler et al., 2006; Nigg et al., 2002;

Ramtekkar et al., 2010).

In patients, the severity of ADHD symptoms declines with age, and it has been shown that HI symptoms decline more rapidly than IA symptoms (Biederman et al., 2000). Nonetheless, ADHD frequently persists from childhood into adulthood, with estimated persistence rates varying between $15 \%$ and over $60 \%$, depending on the definition of persistence (Faraone et al., 2015). A clear difference in the male/female ratio in ADHD cases is observed across the lifespan. In childhood, boys are more likely to be diagnosed with ADHD than girls, with an estimated ratio of 3:1 in population-based studies and of 5:1 to 10:1 in clinical studies (Biederman et al., 2002; Gaub and Carlson, 1997). ADHD in adults shows a more sexbalanced distribution, with male-female ratios from 1:1 to 2:1 (Cortese et al., 2016; Williamson and Johnston, 2015). 
Anxiety disorder is a common comorbidity in individuals with ADHD, both in children and adults. It has been estimated that 35\% of children/adolescents and $47 \%$ of adults with ADHD have at least one comorbid anxiety disorder (Kessler et al., 2006; Schatz and Rostain, 2006). On the other hand, about 15-27\% of adults with an anxiety disorder suffer from ADHD (Mancini et al., 1999; Van Ameringen et al., 2011).

An individual's personality has also shown to be relevant for ADHD's clinical manifestation. Personality is often subdivided into five dimensions (neuroticism, agreeableness, conscientiousness, extraversion and openness), and three of those (low agreeableness, low conscientiousness, and high neuroticism) have shown reliable associations with overall ADHD symptoms score, and IA and/or HI scores individually (Gomez and Corr, 2014; McCrae and John, 1992). In studies using the Eysenck Personality Questionnaire (EPQ), which distinguishes the three dimensions of neuroticism, psychoticism, and extraversion, ADHD symptoms were strongly linked to high neuroticism and high psychoticism scores (Gudjonsson et al., 2009). These associations have been found in adult ADHD patients(Gomez and Corr, 2014), as well as in adolescents with ADHD (Miller et al., 2008). The association between extraversion and ADHD is inconsistent across studies (Gomez and Corr, 2014; Knouse et al., 2013; Nigg et al., 2002). A clinical study showed that hyperactivity predicted higher extraversion, but not impulsivity and inattention (Knouse et al., 2013). Nigg and coworkers, after examining six adult clinical and population samples, concluded that the association between extraversion and ADHD may be limited to self-reports (Nigg et al., 2002).

Many symptoms/characteristics of psychiatric disorders are also observed in healthy individuals (Coghill and Sonuga-Barke, 2012). Such disorder-like traits tend to show a continuous distribution in the general population; with psychiatric cases, including ADHD, clustering at the extreme end (Levy et al., 1997). Arcos-Burgos et al. reported that nearly 60\% of the general population displays symptoms of IA and HI (Arcos-Burgos and Acosta, 2007). Genetic studies also yield support for a continuum of ADHD symptoms in the population (Larsson et al., 2012). Twin studies have shown high heritability estimates for dimensional ADHD symptoms in non-clinical samples (Larsson et al., 2012; Levy et al., 1997). Another study found that a polygenic risk score derived from population ADHD symptoms was associated with ADHD (Stergiakouli et al., 2015). Within the Early genetics and lifecourse Epidemiology (EAGLE) consortium, a genetic correlation estimated at 0.96 was recently reported for genome-wide association study (GWAS) results of ADHD in patients and ADHD symptoms in children/adolescents from the population (Middeldorp et al., 2016). 
Although ADHD symptoms exist in the population, there are few studies reporting on the risk factors for those in the population. If ADHD is the extreme end of a continuum of ADHD symptoms in the population, it stands to reason that associations with anxiety disorder and personality traits observed in patients with ADHD should also be found in general population ADHD symptoms. In this study, we therefore set out to evaluate whether factors affecting clinically diagnosed adult ADHD also associate with ADHD symptoms in adults from the general population. We selected childhood ADHD symptoms, age, sex, anxiety symptoms, and personality traits as factors of interest and investigated whether these are associated with the unique features of each ADHD domain (IA and HI symptoms) in the general population.

\section{Experimental procedures}

\subsection{Participants}

This study was performed in participants from the Nijmegen Biomedical Study (NBS, www.nijmegenbiomedischestudie.nl), a population-based survey conducted by the Radboud university medical center (Galesloot et al., 2017). The study was approved by from the Institutional Review Board. A random sample of 22,451 adult residents of the municipality of Nijmegen, The Netherlands, received an invitation to fill out a postal questionnaire on lifestyle and medical history in 2002 (NBS-1). Of these, 41.6\% $(n=9,350)$ returned the first questionnaire. Participants $(n=7,986)$ who had given permission to be approached for further research (NBS-2), were invited to fill out a second questionnaire in 2005 including questions concerning ADHD symptoms; a total of 4,987 participants filled out the ADHD related questionnaire. Sample sizes for each of the analyses described in this paper may slightly vary, depending on the availability of additional variables included (see Table1). All participants completed written informed consent.

\subsection{Measures}

\subsubsection{ADHD}

ADHD symptoms in the NBS population were measured using the Dutch version of the ADHD DSM-IV rating scale (Kooij et al., 2005), which assesses the 18 DSM-IV items for ADHD. This rating scale assesses, through self-report, current IA symptoms, current HI symptoms, and retrospectively ADHD symptoms in childhood. In order to increase the reliability of self-reports, five of the 18 DSM-IV current symptom items are split and reformulated into 2 items. Therefore, the questionnaire on current symptoms (within the last 6 months) consists of 23 items in total. Three items assess retrospective recall of the presence of 
inattention, hyperactivity, and impulsivity symptoms in childhood at age of 7-8 years (Kooij et al., 2005). Each item was rated on a four-point scale from 0 to 3 ( $0=$ "rarely or never"; 1=“sometimes”; 2=“often”; 3=“very often”). A symptom was regarded as present if the answer given to the item was “often” or “very often” (score 2 or 3). For analysis, the 23 items regarding current symptoms were re-calculated to the original 18 DSM-IV items, where, for the five split items, the symptom was regarded as present if the answer was positive (score 2 or 3) for either one of its parts.

\subsubsection{Anxiety}

Current anxiety symptoms were assessed using the Symptom Check List-90-R (SCL-90-R) anxiety subscale (Derogatis and Savitz, 2000). The SCL-90-R is a widely applied selfassessment instrument for a broad range of psychological problems. The anxiety subscale we applied in NBS consists of ten items, which are rated from zero to four ( $0=$ "not at all”; $1=$ "a little bit”; 2=“moderately”; 3=“quite a bit” 4=“extremely”).We used the total score, with range from zero to 40 , for analysis.

\subsubsection{Personality}

Personality traits were assessed using the Eysenck Personality Questionnaire Revised-Short Form (EPQR-S) (Eysenck et al., 1985). Three main personality dimensions - neuroticism, extraversion, and psychoticism - are assessed by the EPQR-S through 12 binary (yes/no) questions for each of them. Scores for each dimension range from zero to 12, with higher scores indicating a higher tendency to have the representative personality traits. Neuroticism represents emotional instability and stress reactivity; extraversion reflects sociability and positive mood; and psychoticism refers to tough-mindedness, aggression, and ego-centricity (Eysenck, 1990).

\subsection{Statistical Analyses}

Descriptive statistics (means, standard deviations (SDs), proportion, median and interquartile range (Q1-Q3)) of IA and HI symptoms, sex, age, childhood ADHD symptoms, anxiety symptoms, and personality traits were calculated for the total sample and stratified by sex. Ttests (for normally distributed variables), Pearson's Chi-squared test, or Mann-Whitney U tests (for non-normal distributions) were conducted to assess differences between males and females. 
Pearson's Chi-squared test was used to compare the frequency of current IA and HI symptoms between groups defined by retrospectively self-reported number of childhood ADHD symptoms. In these analyses, current IA and HI symptoms were categorized as not present (0 symptoms), below ADHD-threshold (1-4 symptoms), and above ADHD-threshold (5-9 symptoms) for each domain, as the presence of five or more symptoms on at least one of the ADHD domains is one of the diagnostic criteria for ADHD in adults according to DSM-5 (APA, 2013).

Partial Spearman correlation analysis was applied to estimate the relation between the number of current IA/HI symptoms and age, while controlling for sex. We also stratified the sample by the presence of childhood ADHD symptoms in order to test, whether observed differences were similar between groups with and without childhood ADHD symptoms.

To assess how factors known to influence ADHD affect ADHD symptoms in the general population, the Hurdle negative binomial (HNB) model was applied. The HNB model was developed to cope with count data that are over-dispersed and zero-inflated (Zuur et al., 2009). It consists of two sub-models: 1) a binomial logistic model (referred to as zero-model) is used on all observations to predict the probability of having non-zero counts, 2) the negative binomial model (referred to as count-model) is used on the subset of observations with counts $>0$ to predict the number of symptoms. The zero- and count-models were run simultaneously.

In the present study, the statistical distribution of current ADHD symptoms was overdispersed with excessive zeros (IA symptoms: 71.4\% were zero; HI symptoms: $54.9 \%$ were zero; the symptom distributions are shown in Supplementary Figure 2); the characteristics of the data fitted the assumptions for a HNB model. In order to assess the association between current IA/HI symptoms and selected factors in the general population, we used the zeromodel to determine the relation between these factors and the probability of participants reporting at least one IA or HI symptom (analyses were performed separately for each ADHD domain), and we used the count-model to determine the relation between the factors and the number of IA or HI symptoms that participants with at least one symptom reported. Given the known co-occurrence of IA and HI symptoms (APA, 2013), the number of IA or HI symptoms were included as covariate for each other to extract the unique features of each ADHD domain. 
Statistical analyses were conducted using SPSS 22.0 statistical software package (SPSS Inc. Chicago, IL, USA). The HNB analyses were performed using the pscl package in R programming v3.1.1 (Achim. et al., 2008). A Bonferroni-corrected p-value threshold for significance was set at $0.05 / 5=0.0125$. Secondary analyses were performed stratifying by sex.

\section{Results}

\subsection{Participant characteristics}

The NBS sample included in this study consisted of 4,987 participants, of whom 2,682 (53.8\%) were females. The mean age of participants was 56.1 years (SD 16.8, range 21-97 years). Table 1 shows the demographics of the sample. Supplementary Figure 1 shows a sexstratified age distribution of participants; In our sample, the mean age of males was $59.0 \pm 16.5$ years, which was significantly higher than the mean age of females ( $53.6 \pm 16.7$ years; $t=11.5$, $\mathrm{p}<0.0001$ ). Supplementary Figure 2 shows the distribution of current ADHD symptoms. A higher proportion of females than males reported current HI symptoms, but there was no significant difference in current IA symptoms or childhood ADHD symptoms between females and males. Females scored higher on anxiety symptoms and on neuroticism and extraversion personality traits, but lower on psychoticism than males (see Table1).

\subsection{Current IA and HI symptoms and stratification by childhood ADHD symptoms}

From a total of 4747 participants with complete information, 3400 (71.6\%) scored zero, 1228 (25.9\%) scored between 1-4, and 119 (2.5\%) scored 5 or more current IA symptoms. A total of 4778 participants completed the current HI symptom items. Among them, 2626 (55.0\%) scored zero HI symptoms, 2016 (42.2\%) scored between 1-4, and 136 (2.8\%) scored 5 or more HI symptoms. The frequency of current IA and HI symptoms in adulthood stratified by childhood ADHD symptoms is presented in Figure 1. Participants with a higher number of current symptoms for the IA subscale and/or for the HI subscale also reported a significantly higher number of childhood ADHD symptoms (IA: $\chi^{2}=326.3$; $\mathrm{df}=6, \mathrm{p}<0.0001$; $\mathrm{HI}: \chi^{2}=325.0$; $\mathrm{df}=6, \mathrm{p}<0.0001)$.

\subsection{Current IA and HI symptoms over age}

Current HI symptom score was negatively correlated with age $(r=-0.15, p<0.0001$; Figure 2A), while current IA symptoms remained fairly constant over age ( $\mathrm{r}=0.01, \mathrm{p}=0.68$; Figure 2A), after adjustment for sex. A similar picture emerged, when the population was adjusted for the absence/presence of childhood ADHD symptoms (Figure 2B-C). 


\subsection{Simultaneous relation between factors of interest and current IA and HI symptoms}

Results for the association of ADHD symptoms with factors of interest are given in Table 2. For IA symptoms (Table 2a), the HNB regression zero-model, using all data, showed that an increased age, more childhood ADHD symptoms, higher neuroticism scores, and higher psychoticism scores were associated with a higher probability of reporting current IA symptoms, whereas people scoring higher on extraversion were less likely to report current IA symptoms. Also, females were more likely to report current IA symptoms than males. In the count-model, higher neuroticism scores were related to an increase in the number of IA symptoms reported, while higher levels of extraversion were associated with less current IA symptoms. Resutls for childhood ADHD symptoms did not reach significance in the count model. No significant association between anxiety symptoms and IA symptoms was observed in either the zero-model or the count-model. For HI symptoms (Table 2b), the zero-model showed that increasing age decreased the proportion of participants reporting current $\mathrm{HI}$ symptoms. Higher levels of childhood ADHD symptoms, anxiety symptoms, neuroticism, and extraversion were associated with a higher proportion of participants having current $\mathrm{HI}$ symptoms (zero-model) and with a higher number of current HI symptoms (count-model). Scoring high on psychoticism was related to a higher numbers of current HI symptoms in the count-model. No effect of sex on HI symptoms was observed in either the zero-model or the count-model.

\subsection{Differences between sexes}

After stratifying by sex, most of the associations remained the same as in the whole sample, although some effects became less significant, due to the reduced sample size. For IA symptoms, the sex-stratified analysis indicated that the effect of age observed in the overall sample was driven mainly by females (Supplementary Table 1a). An effect on IA symptoms only seen in the sex-stratified analysis was an association with childhood ADHD symptoms in the count model, which was observed in females (Supplementary Table 1a). For HI symptoms, the count model association with childhood ADHD turned out to be male-specific (Supplementary Table 2b). In contrast, females were found to drive the association between adult HI symptoms and anxiety symptom scores (Supplementary Table 2b). 


\section{Discussion}

In this study, we sought to evaluate whether factors known to influence clinically diagnosed adult ADHD are also linked to ADHD symptoms along the continuum of such symptoms in adults from the general population. Indeed, our data show that all factors of interest, i.e. childhood ADHD symptoms, age, sex, anxiety symptoms, and personality traits were associated with current IA or HI symptoms in adults from the general population.

Age was associated with ADHD symptoms in the general population as expected. The partial correlation analyses indicated that IA symptoms remained fairly constant over age, whereas HI symptoms declined with increasing age; this age effect was seen regardless of childhood ADHD symptom history. These findings are in line with clinical studies showing that IA symptoms are more persistent than HI symptoms from childhood into adolescence and (early) adulthood (Biederman et al., 2000; Faraone et al., 2006). In the HNB model analysis, age was associated with a smaller proportion of participants reporting HI symptoms, but it increased the probability of participants to report current IA symptoms. This increased probability of having IA symptoms probably reflects an age-related reduction in attention efficiency in our middle to late adulthood sample (de Fockert et al., 2009). The reduction of attention capability may be due to inhibition deficits, which involve age-associated decline in neural function (Haring et al., 2013). Our results indicate that the continuum of ADHD symptoms in the general population follows a similar pattern as previously reported in patients, IA symptoms remain stable and HI symptoms decrease with age, and this pattern continues into later stages of life. In addition, we may also have picked-up first signs of cognitive decline with the HNB model, given the age of our sample.

For sex, our unadjusted analysis showed that more females had HI symptoms; in the HNB model, in which the effects of age and other factors had been controlled, we also found that female sex was associated with the likelihood of reporting IA symptoms (Laura Ghirardi et al.). This association was validated by the sex-stratified analysis. Our result is not well comparable with earlier population studies, which have concentrated on the severity of symptoms. In children, higher symptom scores have been reported in males (Arnett et al., 2015; Pinto et al., 2016), but the differences between the sexes seem to become less pronounced later in life; where studies have reported higher symptom levels in both males and females (Ghirardi et al., Submitted; Polderman et al., 2014). A similar trend is seen in patients with ADHD which a male preponderance of the disorder normalizes towards adulthood 
(Faraone et al., 2015). Additional effects of age were observed in the sex-stratified analyses. As what may have been expected, associations of childhood ADHD symptoms with IA symptoms were observed in females in the count model; and those with HI symptoms were seen in males. However, in general most associations observed in the total sample were shared among males and females.

Childhood ADHD symptoms increased the likelihood of participants to report current adult IA and HI symptoms as well as the number of these symptoms reported in the HNB model. Consistent with this, the Pearson's Chi-squared tests showed that participants with higher current ADHD symptoms were inclined to report more ADHD symptoms in childhood. Numerous follow-up studies have demonstrated that a proportion of individuals with ADHD in childhood continues to experience symptoms in adolescence and (early) adulthood (Rasmussen and Levander, 2009). Genetic factors that aggregated in a polygenic risk score for ADHD contribute to the persistence of ADHD symptoms from childhood to adolescence in the general population (Lara et al., 2009). Treating ADHD symptoms as a continuum in the general population, our results confirmed that the same pattern of correlation between ADHD symptoms in childhood and adulthood exists in the general population as in clinical samples, and that this pattern is also seen in middle to late adulthood. We found that anxiety symptoms in our population-based sample increased the likelihood and the number of HI symptoms participants reported. This association was drived by females. No significant association with IA symptoms was seen. Previously, childhood ADHD patients with comorbid anxiety disorders were shown to be more likely to display IA symptoms than HI symptoms compared to patients only with ADHD (Riglin et al., 2016); and females with ADHD tend to have more internalizing problems, such as anxiety and depression (Quinn and Madhoo, 2014). However, there is little literature on the relationship between adult ADHD symptom domains and anxiety. A recent study reported that the severity of ADHD symptoms is associated with social anxiety symptoms among university students (Newcorn et al., 2001). Our findings support a link between ADHD symptoms and anxiety in the general population, and implicate HI symptoms in particular.

Regarding personality traits, our results indicated that higher neuroticism scores were significantly associated with increased adult IA and HI symptoms in the general population. This finding is in line with results of a meta-analysis referring to the specific ADHD domain scores, which indicated that neuroticism is linked to both IA and HI in clinical samples and (though with somewhat smaller effects) in community samples (Gomez and Corr, 2014). For 
extraversion, we found opposite links to current IA and HI symptoms: this personality trait was linked to decreased IA and to increased HI symptoms. The existing literature on the association between extraversion and ADHD is not consistent. A previous population study in adults showed that undergraduates with high ADHD symptom counts had higher extraversion scores compared to individuals with low ADHD symptoms (Braaten and Rosén, 1997). Another study found that extraversion was a significant predictor of the predominantly $\mathrm{HI}$ subtype of ADHD, but not the predominantly IA subtype (Parker et al., 2004). A third study reported no difference in extraversion between university students with probable ADHD and those without ADHD (Rowe et al., 2007). Our study corroborates the findings for extraversion linking to $\mathrm{HI}$ in two of the studies, and additionally presents evidence for an opposite relation with IA symptoms. A possible mechanism behind this finding might be that high extraversion is connected to loosened inhibitory control resulting in increased attentional performance (Rowe et al., 2007), as an earlier study found that extraversion was linked to increased attention performance during a change detection task (Hahn et al., 2015). In addition to findings for neuroticism and extraversion, we also found that psychoticism was positively related to both IA and HI symptoms in the adult population. In this, our data are in line with a meta-analysis, which grouped psychoticism from EPQ and agreeableness from the Big Five Model into 'agreeable inhibition', and reported a significant positive association between this trait and ADHD symptoms (both IA and HI) in clinical and community samples (Gomez and Corr, 2014).

This work has strengths and limitations that should be acknowledged. As often observed in psychiatric research in the general population, the distribution of outcomes in our study was often positively skewed and inflated at zero. More conventional statistical models, which require normally distributed residuals, provide a poor fit for such data and may lead to incorrect conclusions. We therefore used the HNB model, which provides the advantage of using two sub-models, one investigating the probability of non-zero scores, and the second investigating the distribution of non-zero scores. We found that several effects were seen in both models, which indicate that age, sex, childhood ADHD, anxiety symptoms, and personality traits influence the whole dimension of ADHD symptoms in the adult population. As another strength, this study used data from the NBS, a population-based study set-up with the aim of obtaining a universal reference population and for studying population traits. It has large sample size and covers a broad age range (21-97 years), which provides a good opportunity to examine the natural distribution of ADHD symptomatology across the lifespan. 
However, we did not have information on diagnostic status for ADHD in childhood and for the participants with more than five current IA/HI symptoms. We kept these participants, who were probable-ADHD patients in the analysis; which may have caused some bias. However, this being only a small precentage, we do not expect that it influenced our results. Another limitation is that ADHD symptoms were assessed with a self-report questionnaire without information on impairment, and childhood symptoms were assessed by retrospective recall. Such data may be subjects to recall bias.

In this study, we did observe relatively strong associations among anxiety, personality traits, and self-reported adult ADHD symptoms. An important point to be investigated in the future based on those findings is the direction of effects and the underlying mechanisms. An earlier study in Turkish university students reported that extraversion and neuroticism affected the association between ADHD symptoms and anxiety symptoms, suggesting mediation by the personality factors (Riglin et al., 2016). Longitudinal studies would be optimal to investigate causal relationships amongst the phenotypes in the future. Moreover, using the raw scores of ADHD questionnaire instead of the ADHD symptoms would lead a better presentation of the trait nature of ADHD in the general population.

In conclusion, our data suggest that ADHD symptoms in the general population are influenced by childhood ADHD symptoms, age, sex, and personality traits, just like the clinical diagnosis of ADHD. This strengthens the evidence that ADHD symptoms in the population have similar relationships with other phenotypes as the clinical extreme of the continuum - also in adults. An interesting future prospect arising from these findings is the possibility to perform gene-finding studies for ADHD in population samples, which are easier to collect in larger numbers than clinical samples.

\section{Appendix A. Supporting information}

Supplementary data associated with this article can be found in supplementary files.

\section{Acknowledgements}

We would like to thank all the participants. 


\section{Contributors}

Barbara Frank and Alejandro AriasVasquez contributed to design the study and statistical analyses. Ting Li undertook the analyses and wrote the first draft of the manuscript with contribution from Nina Roth Mota. All co-authors contributed to critically review and approve the final manuscript.

\section{Role of funding source}

The Nijmegen Biomedical Study (NBS) was financed by the participating Radboudumc departments and a small investment grant from the Radboudumc Executive Board. The municipality Nijmegen and the community health service Nijmegen contributed funding. Ting $\mathrm{Li}$ is supported by China Scholarship Council (CSC) under the Grant CSC n²01507720006. Nina Roth Mota is supported by funding from National Institute of Mental Health (R01MH106655) and the European Community's Horizon 2020 Research and Innovation Program under grant agreements ${ }^{\circ} 667302$ (CoCA). Barbara Franke and her team are supported by funding from a personal Vici grant of the Netherlands Organisation for Scientific Research (NWO; grant 016-130-669, to BF), from the European Community's Seventh Framework Programme (FP7/2007-2013) under grant agreements n602805 (Aggressotype), $\mathrm{n}^{\circ} 602450$ (IMAGEMEND), and $\mathrm{n}^{\circ} 278948$ (TACTICS), and from the European Community's Horizon 2020 Programme (H2020/2014-2020) under grant agreements $n^{\circ} 643051$ (MiND) and $n^{\circ} 667302$ (CoCA). In addition, this work was supported by the European College of Neuropsychopharmacology (ECNP Network “ADHD across the Lifespan”).

\section{Conflict of Interest}

Barbara Franke has received educational speaking fees from Shire and Medice. In the past 3 years, Jan Buitelaar has been a consultant to/member of advisory board of/and/or speaker for Janssen Cilag BV, Eli Lilly, Lundbeck, Shire, Roche, Medice, Novartis, and Servier. He has received research support from Roche and Vifor. He is not an employee of any of these companies, and not a stock shareholder of any of these companies. He has no other financial or material support, including expert testimony, patents, royalties. The other authors declare no conflict of interest. 


\section{References}

Achim., Z., Christian., K., Simon., J., 2008. Regression Models for Count Data in R. Journal of Statistical Software. 27.

APA, 2013. Diagnostic and Statistical Manual of Mental Disorders, 5th edition. Arlington, VA. American Psychiatric Association.

Arcos-Burgos, M., Acosta, M.T., 2007. Tuning major gene variants conditioning human behavior: the anachronism of ADHD. Current opinion in genetics \& development 17, 234238.

Arnett, A.B., Pennington, B.F., Willcutt, E.G., DeFries, J.C., Olson, R.K., 2015. Sex differences in ADHD symptom severity. Journal of child psychology and psychiatry, and allied disciplines 56, 632-639.

Biederman, J., Mick, E., Faraone, S.V., 2000. Age-dependent decline of symptoms of attention deficit hyperactivity disorder: impact of remission definition and symptom type. The American journal of psychiatry 157, 816-818.

Biederman, J., Mick, E., Faraone, S.V., Braaten, E., Doyle, A., Spencer, T., Wilens, T.E., Frazier, E., Johnson, M.A., 2002. Influence of gender on attention deficit hyperactivity disorder in children referred to a psychiatric clinic. The American journal of psychiatry 159, 36-42. Braaten, E.B., Rosén, L.A., 1997. Emotional reactions in adults with symptoms of attention deficit hyperactivity disorder. Personality and Individual Differences 22, 355-361.

Coghill, D., Sonuga-Barke, E.J., 2012. Annual research review: categories versus dimensions in the classification and conceptualisation of child and adolescent mental disorders-implications of recent empirical study. Journal of child psychology and psychiatry, and allied disciplines 53, 469-489.

Cortese, S., Faraone, S.V., Bernardi, S., Wang, S., Blanco, C., 2016. Gender differences in adult attention-deficit/hyperactivity disorder: results from the National Epidemiologic Survey on Alcohol and Related Conditions (NESARC). The Journal of clinical psychiatry 77, e421-428.

Costello, E.J., Mustillo, S., Erkanli, A., Keeler, G., Angold, A., 2003. Prevalence and development of psychiatric disorders in childhood and adolescence. Archives of general psychiatry 60, 837-844.

de Fockert, J.W., Ramchurn, A., van Velzen, J., Bergstrom, Z., Bunce, D., 2009. Behavioral and ERP evidence of greater distractor processing in old age. Brain research 1282, 67-73.

Derogatis, L.R., Savitz, K.L.M., Mark E., 2000. The SCL-90-R and Brief Symptom Inventory (BSI) in primary care. Handbook of psychological assessment in primary care settings. Mahwah, NJ, US: Lawrence Erlbaum Associates Publishers.

Eysenck, H.J., 1990. Genetic and environmental contributions to individual differences: the three major dimensions of personality. Journal of personality 58, 245-261.

Eysenck, S.B.G., Eysenck, H.J., Barrett, P., 1985. A revised version of the psychoticism scale Personality and Individual Differences 6, 21-29.

Faraone, S.V., Asherson, P., Banaschewski, T., Biederman, J., Buitelaar, J.K., Ramos-Quiroga, J.A., Rohde, L.A., Sonuga-Barke, E.J., Tannock, R., Franke, B., 2015. Attentiondeficit/hyperactivity disorder. Nature reviews. Disease primers 1, 15020. 
Faraone, S.V., Biederman, J., Mick, E., 2006. The age-dependent decline of attention deficit hyperactivity disorder: a meta-analysis of follow-up studies. Psychological medicine 36, 159165.

Galesloot, T.E., Vermeulen, S.H., Swinkels, D.W., de Vegt, F., Franke, B., den Heijer, M., de Graaf, J., Verbeek, A.L., Kiemeney, L.A., 2017. Cohort Profile: The Nijmegen Biomedical Study (NBS). International journal of epidemiology.

Gaub, M., Carlson, C.L., 1997. Gender differences in ADHD: a meta-analysis and critical review. Journal of the American Academy of Child and Adolescent Psychiatry 36, 1036-1045. Ghirardi, L., Pettersson, E., Taylor, M.J., Freitag, C.M., Franke, B., Asherson, P., Larsson, H., Kuja-Halkola, R., Submitted. Genetic and environmental contribution to the overlap between ADHD and ASD dimensions in adults: a twin study. .

Gomez, R., Corr, P.J., 2014. ADHD and personality: a meta-analytic review. Clinical psychology review 34, 376-388.

Gudjonsson, G.H., Sigurdsson, J.F., Young, S., Newton, A.K., Peersen, M., 2009. Attention Deficit Hyperactivity Disorder (ADHD). How do ADHD symptoms relate to personality among prisoners? Personality and Individual Differences 47, 64-68.

Hahn, S., Buttaccio, D.R., Hahn, J., Lee, T., 2015. Personality and attention: Levels of neuroticism and extraversion can predict attentional performance during a change detection task. Quarterly journal of experimental psychology (2006) 68, 1041-1048.

Haring, A.E., Zhuravleva, T.Y., Alperin, B.R., Rentz, D.M., Holcomb, P.J., Daffner, K.R., 2013. Age-related differences in enhancement and suppression of neural activity underlying selective attention in matched young and old adults. Brain research 1499, 69-79. Kessler, R.C., Adler, L., Barkley, R., Biederman, J., Conners, C.K., Demler, O., Faraone, S.V., Greenhill, L.L., Howes, M.J., Secnik, K., Spencer, T., Ustun, T.B., Walters, E.E., Zaslavsky, A.M., 2006. The prevalence and correlates of adult ADHD in the United States: Results from the National Comorbidity Survey Replication. Am J Psychiat 163, 716-723.

Knouse, L.E., Traeger, L., O'Cleirigh, C., Safren, S.A., 2013. Adult attention deficit hyperactivity disorder symptoms and five-factor model traits in a clinical sample: a structural equation modeling approach. The Journal of nervous and mental disease 201, 848-854. Kooij, J.J., Buitelaar, J.K., van den Oord, E.J., Furer, J.W., Rijnders, C.A., Hodiamont, P.P., 2005. Internal and external validity of attention-deficit hyperactivity disorder in a population-based sample of adults. Psychological medicine 35, 817-827.

Lara, C., Fayyad, J., de Graaf, R., Kessler, R.C., Aguilar-Gaxiola, S., Angermeyer, M., Demytteneare, K., de Girolamo, G., Haro, J.M., Jin, R., Karam, E.G., Lepine, J.P., Mora, M.E.M., Ormel, J., Posada-Villa, J., Sampson, N., 2009. Childhood Predictors of Adult Attention-Deficit/Hyperactivity Disorder: Results from the World Health Organization World Mental Health Survey Initiative. Biological psychiatry 65, 46-54.

Larsson, H., Anckarsater, H., Rastam, M., Chang, Z., Lichtenstein, P., 2012. Childhood attention-deficit hyperactivity disorder as an extreme of a continuous trait: a quantitative genetic study of 8,500 twin pairs. Journal of child psychology and psychiatry, and allied disciplines 53, 73-80.

Laura Ghirardi, Erik Pettersson, Paul Lichtenstein, J, T.M., Freitag, C.M., Franke, B., Henrik Larsson, Kuja-Halkola, R., Genetic and environmental contribution to the overlap between ADHD and ASD dimensions in adults: a twin study. (Submitted).

Levy, F., Hay, D.A., McStephen, M., Wood, C., Waldman, I., 1997. Attention-deficit hyperactivity disorder: a category or a continuum? Genetic analysis of a large-scale twin study. Journal of the American Academy of Child and Adolescent Psychiatry 36, 737-744. 
Mancini, C., Van Ameringen, M., Oakman, J.M., Figueiredo, D., 1999. Childhood attention deficit/hyperactivity disorder in adults with anxiety disorders. Psychological medicine 29, 515-525.

McCrae, R.R., John, O.P., 1992. An introduction to the five-factor model and its applications. Journal of personality 60, 175-215.

Middeldorp, C.M., Hammerschlag, A.R., Ouwens, K.G., Groen-Blokhuis, M.M., Pourcain, B.S., Greven, C.U., Pappa, I., Tiesler, C.M.T., Ang, W., Nolte, I.M., Vilor-Tejedor, N., Bacelis, J., Ebejer, J.L., Zhao, H., Davies, G.E., Ehli, E.A., Evans, D.M., Fedko, I.O., Guxens, M., Hottenga, J.J., Hudziak, J.J., Jugessur, A., Kemp, J.P., Krapohl, E., Martin, N.G., Murcia, M., Myhre, R., Ormel, J., Ring, S.M., Standl, M., Stergiakouli, E., Stoltenberg, C., Thiering, E., Timpson, N.J., Trzaskowski, M., van der Most, P.J., Wang, C., Nyholt, D.R., Medland, S.E., Neale, B., Jacobsson, B., Sunyer, J., Hartman, C.A., Whitehouse, A.J.O., Pennell, C.E., Heinrich, J., Plomin, R., Smith, G.D., Tiemeier, H., Posthuma, D., Boomsma, D.I., 2016. A Genome-Wide Association Meta-Analysis of Attention-Deficit/Hyperactivity Disorder Symptoms in Population-Based Pediatric Cohorts. J Am Acad Child Adolesc Psychiatry 55, 896-905.e896. Miller, C.J., Miller, S.R., Newcorn, J.H., Halperin, J.M., 2008. Personality characteristics associated with persistent ADHD in late adolescence. Journal of abnormal child psychology 36, 165-173.

Newcorn, J.H., Halperin, J.M., Jensen, P.S., Abikoff, H.B., Arnold, L.E., Cantwell, D.P., Conners, C.K., Elliott, G.R., Epstein, J.N., Greenhill, L.L., Hechtman, L., Hinshaw, S.P., Hoza, B., Kraemer, H.C., Pelham, W.E., Severe, J.B., Swanson, J.M., Wells, K.C., Wigal, T., Vitiello, B., 2001. Symptom profiles in children with ADHD: effects of comorbidity and gender. Journal of the American Academy of Child and Adolescent Psychiatry 40, 137-146.

Nigg, J.T., John, O.P., Blaskey, L.G., Huang-Pollock, C.L., Willcutt, E.G., Hinshaw, S.P., Pennington, B., 2002. Big five dimensions and ADHD symptoms: links between personality traits and clinical symptoms. J Pers Soc Psychol 83, 451-469.

Parker, J.D.A., Majeski, S.A., Collin, V.T., 2004. ADHD symptoms and personality: relationships with the five-factor model. Personality and Individual Differences 36, 977-987. Pinto, R., Rijsdijk, F., Ronald, A., Asherson, P., Kuntsi, J., 2016. The Genetic Overlap of Attention-Deficit/Hyperactivity Disorder and Autistic-like Traits: an Investigation of Individual Symptom Scales and Cognitive markers. Journal of abnormal child psychology 44, 335-345. Polanczyk, G., de Lima, M.S., Horta, B.L., Biederman, J., Rohde, L.A., 2007. The worldwide prevalence of ADHD: a systematic review and metaregression analysis. The American journal of psychiatry 164, 942-948.

Polderman, T.J., Hoekstra, R.A., Posthuma, D., Larsson, H., 2014. The co-occurrence of autistic and ADHD dimensions in adults: an etiological study in 17,770 twins. Translational psychiatry 4, e435.

Quinn, P.O., Madhoo, M., 2014. A review of attention-deficit/hyperactivity disorder in women and girls: uncovering this hidden diagnosis. Prim Care Companion CNS Disord 16. Ramtekkar, U.P., Reiersen, A.M., Todorov, A.A., Todd, R.D., 2010. Sex and age differences in attention-deficit/hyperactivity disorder symptoms and diagnoses: implications for DSM-V and ICD-11. Journal of the American Academy of Child and Adolescent Psychiatry 49, 217228 e211-213.

Rasmussen, K., Levander, S., 2009. Untreated ADHD in adults: are there sex differences in symptoms, comorbidity, and impairment? Journal of attention disorders 12, 353-360.

Riglin, L., Collishaw, S., Thapar, A.K., Dalsgaard, S., Langley, K., Smith, G.D., Stergiakouli, E., Maughan, B., O'Donovan, M.C., Thapar, A., 2016. Association of Genetic Risk Variants With 
Attention-Deficit/Hyperactivity Disorder Trajectories in the General Population. JAMA psychiatry 73, 1285-1292.

Rowe, G., Hirsh, J.B., Anderson, A.K., 2007. Positive affect increases the breadth of attentional selection. Proc Natl Acad Sci U S A 104, 383-388.

Schatz, D.B., Rostain, A.L., 2006. ADHD with comorbid anxiety: a review of the current literature. Journal of attention disorders 10, 141-149.

Simon, V., Czobor, P., Balint, S., Meszaros, A., Bitter, I., 2009. Prevalence and correlates of adult attention-deficit hyperactivity disorder: meta-analysis. The British journal of psychiatry : the journal of mental science 194, 204-211.

Stergiakouli, E., Martin, J., Hamshere, M.L., Langley, K., Evans, D.M., St Pourcain, B., Timpson, N.J., Owen, M.J., O'Donovan, M., Thapar, A., Davey Smith, G., 2015. Shared genetic influences between attention-deficit/hyperactivity disorder (ADHD) traits in children and clinical ADHD. Journal of the American Academy of Child and Adolescent Psychiatry 54, 322327.

Thomas, R., Sanders, S., Doust, J., Beller, E., Glasziou, P., 2015. Prevalence of attentiondeficit/hyperactivity disorder: a systematic review and meta-analysis. Pediatrics 135, e9941001.

Van Ameringen, M., Mancini, C., Simpson, W., Patterson, B., 2011. Adult attention deficit hyperactivity disorder in an anxiety disorders population. CNS neuroscience $\&$ therapeutics 17, 221-226.

WHO, 1992. International statistical classification of diseases and related health problems, 10th revision. Geneva: World Health Organization.

Williamson, D., Johnston, C., 2015. Gender differences in adults with attentiondeficit/hyperactivity disorder: A narrative review. Clinical psychology review 40, 15-27. Zuur, A.F., leno, E.N., Walker, N.J., Saveliev, A.A., Smith, G.M., 2009. Zero-Truncated and Zero-Inflated Models for Count Data. Mixed effects models and extensions in ecology with R., 261-293. 
Table 1. Characteristics of the NBS sample.

\begin{tabular}{|c|c|c|c|c|c|c|c|c|}
\hline Variables & \multicolumn{2}{|c|}{ Total sample } & \multicolumn{2}{|r|}{ Males } & \multicolumn{2}{|c|}{ Females } & & \\
\hline ADHD & $\mathbf{N}$ & Percentage & $\mathbf{N}$ & Percentage & $\mathbf{N}$ & Percentage & Chi-square & P-value \\
\hline Current IA symptoms & 4885 & & 2273 & $46.5 \%$ & 2612 & $53.5 \%$ & 2.8 & 0.51 \\
\hline 0 symptoms (\%) & 3490 & $71.4 \%$ & 1607 & $70.7 \%$ & 1883 & $72.1 \%$ & & \\
\hline 1-4 symptoms (\%) & 1272 & $26.0 \%$ & 614 & $27.0 \%$ & 658 & $25.2 \%$ & & \\
\hline$>=5$ symptoms (\%) & 123 & $2.5 \%$ & 52 & $2.3 \%$ & 71 & $2.7 \%$ & & \\
\hline Current HI symptoms & 4917 & & 2277 & $46.3 \%$ & 2640 & $53.7 \%$ & 14.8 & 0.001 \\
\hline 0 symptoms (\%) & 2698 & $54.9 \%$ & 1304 & $57.3 \%$ & 1394 & $52.8 \%$ & & \\
\hline 1-4 symptoms (\%) & 2078 & $42.3 \%$ & 924 & $40.6 \%$ & 1154 & $43.7 \%$ & & \\
\hline >=5 symptoms (\%) & 141 & $2.9 \%$ & 49 & $2.2 \%$ & 92 & $3.5 \%$ & & \\
\hline Childhood ADHD symptoms & 4840 & & 2230 & $46.1 \%$ & 2610 & $53.9 \%$ & 3.2 & 0.08 \\
\hline 0 symptoms (\%) & 3710 & $76.7 \%$ & 1690 & $75.8 \%$ & 2020 & $77.4 \%$ & & \\
\hline 1symptoms (\%) & 699 & $14.4 \%$ & 325 & $14.6 \%$ & 374 & $14.3 \%$ & & \\
\hline 2 symptoms (\%) & 277 & $5.7 \%$ & 136 & $6.1 \%$ & 141 & $5.4 \%$ & & \\
\hline 3 symptoms (\%) & 154 & $3.2 \%$ & 79 & $3.5 \%$ & 75 & $2.9 \%$ & & \\
\hline Age and Personality traits & $\mathbf{N}$ & Mean (SD) & $\mathbf{N}$ & Mean (SD) & $\mathbf{N}$ & Mean (SD) & t-test & P-value \\
\hline Age (years) & 4987 & $56.1(16.8)$ & 2305 & $59.0(16.5)$ & 2682 & $53.6(16.7)$ & -0.8 & $<0.0001$ \\
\hline Neuroticism score & 4987 & $3.5(2.9)$ & 2305 & $2.9(2.6)$ & 2682 & $4.1(3.0)$ & -15.1 & $<0.0001$ \\
\hline Extraversion score & 4766 & $7.0(3.2)$ & 2153 & $6.7(3.4)$ & 2499 & $7.4(3.1)$ & -7.3 & $<0.0001$ \\
\hline Anxiety & $\mathbf{N}$ & Median (Q1, Q3) & $\mathbf{N}$ & Median (Q1, Q3) & $\mathbf{N}$ & Median (Q1, Q3) & Mann-Whitney & P-value \\
\hline Anxiety symptoms & 4918 & $1.0(1.0,2.0)$ & 2285 & $0.0(0.0,2.0)$ & 2633 & $1.0(1.0,3.0)$ & -11.2 & $<0.0001$ \\
\hline
\end{tabular}


Table 2a. Hurdle negative binomial regression analysis of current IA symptoms in adulthood $(n=4232 *)$

\begin{tabular}{|c|c|c|c|c|c|c|}
\hline \multirow[b]{2}{*}{ Predictor } & \multicolumn{3}{|c|}{ Zero-model } & \multicolumn{3}{|c|}{ Count-model } \\
\hline & Estimate(SE) & $95 \% \mathrm{CI}$ & $\mathbf{p}$ & Estimate(SE) & $95 \%$ CI & $\mathbf{p}$ \\
\hline Age & $0.01(0.002)$ & $0.003-0.01$ & 0.001 & $0.001(0.002)$ & $-0.003-0.01$ & 0.62 \\
\hline $\operatorname{Sex}^{\mathrm{a}}$ & $-0.26(0.08)$ & $-0.42-0.09$ & 0.002 & $-0.11(0.08)$ & $-0.27--0.05$ & 0.19 \\
\hline $\begin{array}{c}\text { Childhood ADHD } \\
\text { symptoms }\end{array}$ & $0.40(0.05)$ & $0.29-0.50$ & $<.0001$ & $0.08(0.04)$ & 0.003-0.16 & 0.04 \\
\hline Anxiety symptoms & $0.02(0.02)$ & $-0.01-0.05$ & 0.30 & $0.02(0.01)$ & $-0.01-0.04$ & 0.13 \\
\hline Neuroticism & $0.13(0.02)$ & $0.10-0.17$ & $<.0001$ & $0.06(0.02)$ & 0.03-0.09 & $<.0001$ \\
\hline Extraversion & $-0.07(0.01)$ & $-0.10--0.05$ & $<.0001$ & $-0.04(0.01)$ & $-0.06--0.01$ & 0.003 \\
\hline Psychoticism & $0.15(0.02)$ & $0.10-0.20$ & $<.0001$ & $0.02(0.02)$ & $-0.03-0.06$ & 0.49 \\
\hline HI symptoms & $0.62(0.04)$ & $0.55-0.69$ & $<.0001$ & $0.23(0.02)$ & $0.18-0.27$ & $<.0001$ \\
\hline
\end{tabular}

Note. Significant differences are indicated in bold. Zero-model was used to predict the probability of having IA/HI symptoms, count-model predicted the number of IA/HI symptoms. HI symptoms were included as covariate.* Number of participants with information on all variables; ${ }^{\text {a }}$ Sex: effects for males as compared to females. 
Table 2b. Hurdle negative binomial regression analysis of current HI symptoms in adulthood $(n=4232 *)$

\begin{tabular}{|c|c|c|c|c|c|c|}
\hline \multirow[b]{2}{*}{ Predictor } & \multicolumn{3}{|l|}{ Zero-model } & \multicolumn{3}{|c|}{ Count-model } \\
\hline & Estimate(SE) & $95 \% \mathrm{CI}$ & $\mathbf{p}$ & Estimate(SE) & $95 \% \mathrm{CI}$ & $\mathbf{p}$ \\
\hline Age & $-0.02(0.002)$ & $-0.02--0.01$ & $<.0001$ & $-0.002(0.001)$ & $-0.01-0.001$ & 0.26 \\
\hline $\operatorname{Sex}^{a}$ & $-0.12(0.07)$ & $-0.26-0.03$ & 0.12 & $-0.03(0.05)$ & $-0.13-0.07$ & 0.53 \\
\hline $\begin{array}{l}\text { Childhood ADHD } \\
\text { symptoms }\end{array}$ & $0.34(0.05)$ & $0.24-0.44$ & $<.0001$ & $0.09(0.03)$ & $0.04-0.14$ & $<.0001$ \\
\hline Anxiety symptoms & $0.05(0.02)$ & $0.01-0.08$ & 0.004 & $0.02(0.01)$ & $0.01-0.03$ & 0.0001 \\
\hline Neuroticism & $0.20(0.02)$ & $0.16-0.23$ & $<.0001$ & $0.09(0.01)$ & $0.07-0.11$ & $<.0001$ \\
\hline Extraversion & $0.06(0.01)$ & $0.03-0.08$ & $<.0001$ & $0.05(0.01)$ & 0.04-0.07 & $<.0001$ \\
\hline Psychoticism & $-0.003(0.02)$ & $-0.05-0.04$ & 0.88 & $0.05(0.01)$ & 0.03-0.08 & 0.0002 \\
\hline IA symptoms & $0.67(0.05)$ & $0.58-0.77$ & $<.0001$ & $0.16(0.01)$ & $0.13-0.18$ & $<.0001$ \\
\hline
\end{tabular}

Note. Significant differences are indicated in bold. Zero-model was used to predict the probability of having IA/HI symptoms, count-model predicted the number of IA/HI symptoms. IA symptoms were included as covariate. ${ }^{*}$ Number of participants with information on all variables; ${ }^{a}$ Sex: effects for males as compared to females. 
Figure 1. Current ADHD symptoms stratified by presence of childhood ADHD symptoms. A) Current IA symptoms; B) Current HI symptoms.

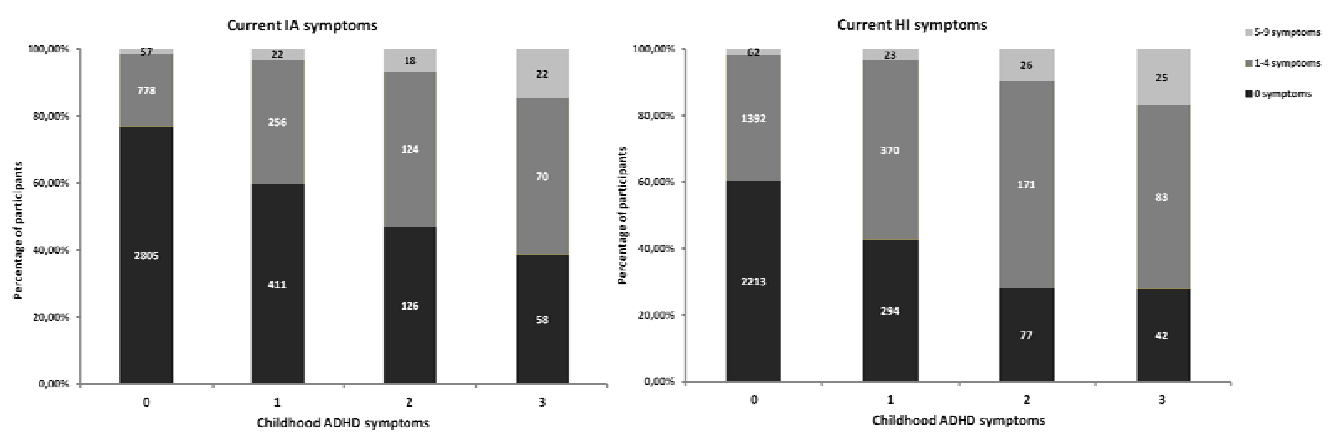


Figure 2. Current ADHD symptoms across age in the NBS population sample, with and without stratification by the number of retrospective childhood ADHD symptoms: A) Any number ofchildhood symptoms ( 0 -3 symptoms; $n=4,885$ for IA, $n=4,914$ for HI); B) no childhood symptoms ( 0 symptoms; $n=3,640$ for IA, $n=3,667$ for HI); C) at least one childhood symptom (1-3 symptoms; $n=1,107$ for IA, $n=1,111$ for HI). Note: Age bin and mean were used to present the results. The correlation ( $\mathrm{r}$ ) and P-value were determined for age in years.
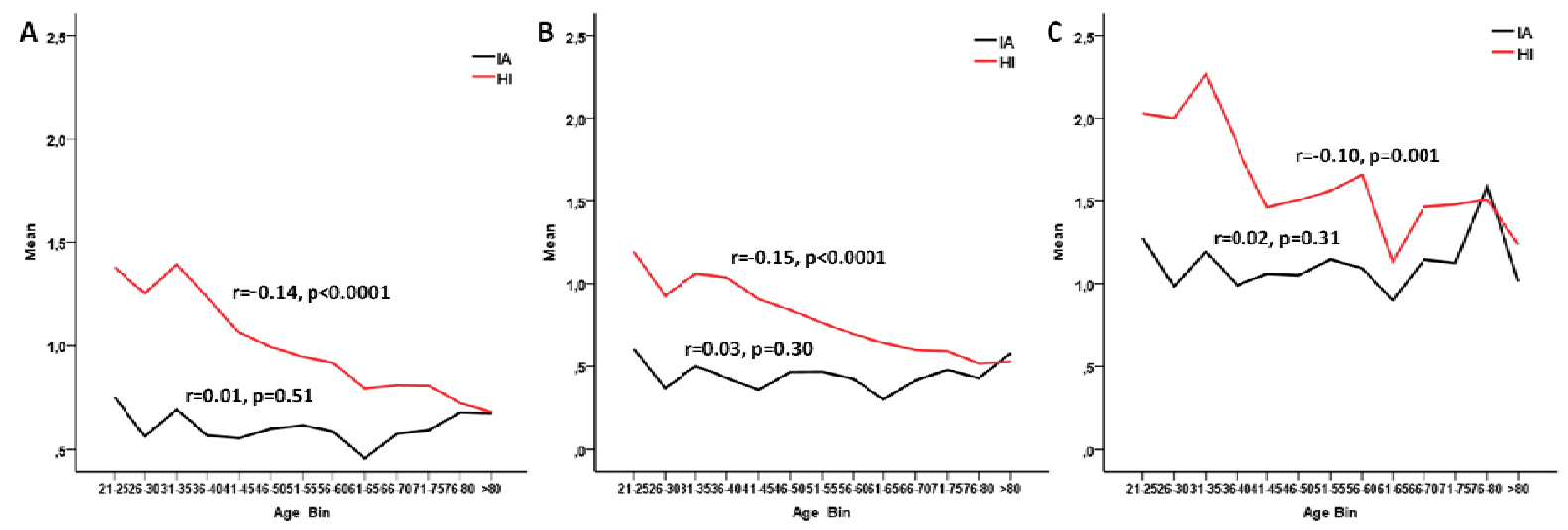
Supplementary Figure 1: The age distribution of participants with sex stratification. Note: Age bin was used to present the results.

Supplementary Figure 2. The distribution of current IA and HI symptoms in the NBS population sample.

Supplementary Table 1a Hurdle negative binomial regression analysis of current IA symptoms in males $(n=1981)$

Supplementary Table 1b Hurdle negative binomial regression analysis of current IA symptoms in females $(n=2251)$

Supplementary Table 2a Hurdle negative binomial regression analysis of current HI symptoms in males $(n=1981)$

Supplementary Table $\mathbf{2 b}$ Hurdle negative binomial regression analysis of current HI symptoms in females $(n=2251)$ 